\title{
Capacity of Vehicular Ad Hoc Networks Based on Multibeam Directional Transmission
}

\author{
Yuhua Wang $\mathbb{D}^{\mathbb{D}}$, Laixian Peng $\mathbb{D}$, and Renhui Xu \\ Army Engineering University of PLA, Nanjing 210000, China \\ Correspondence should be addressed to Laixian Peng; lxpeng@hotmail.com
}

Received 9 August 2021; Revised 30 September 2021; Accepted 9 October 2021; Published 26 October 2021

Academic Editor: Renchao Xie

Copyright (c) 2021 Yuhua Wang et al. This is an open access article distributed under the Creative Commons Attribution License, which permits unrestricted use, distribution, and reproduction in any medium, provided the original work is properly cited.

\begin{abstract}
The development of multibeam directional transmission technology used in vehicular ad hoc networks is drawing much more attention in recent years due to its wider coverage ability than omnidirectional transmission. In this paper, we analyse the transport capacity of the vehicular network using different antenna modes in the transmitter and receiver end, respectively. We first construct the cross-layer model comprising the characteristic of the directional antenna model, arbitrary network model, and interference model. Then, based on scaling laws, we calculate the upper and lower bound of the network capacity with and without the directional multibeam transmission technology. In order to reduce the capacity lower bound computation complexity, several topology frameworks are constructed while taking various interferences into account included in the actual project. Finally, we analyse the capacity under changes of different parameters and also evaluate the law of capacity changes to discover how much improvement multibeam transmission technology can bring to the network performance. Analysis shows that compared with DTOR and OTDR mode, DTDR mode can continue to increase network capacity by 2 to 3 times on the basis of the above two modes.
\end{abstract}

\section{Introduction}

Network capacity is one of the most important indicators to evaluate vehicular ad hoc network (VANET) communication performance. It can provide theoretical support for the technical development of wireless network communication and transmission. Given any set of successful transmissions taking place over time and space, let us say that the network transports one bit-meter when one bit has been transported a distance of one meter toward its destination. Summing all products of bits and the distances over which they are carried is a valuable indicator of a network's transport capacity. Nodes in the VANET are often mobile, and their communication links have time-varying characteristics; therefore, its network topology is unstable. Due to the nature of wireless transmission, the capacity of a wireless network is strictly limited by the number of nodes per unit area and the channel transmission bandwidth. Since Gupta and Kumar [1] proposed the investigating method of network capacity in wireless ad hoc networks based on omnidirectional transmission, many researches have focused on this field. They proposed that the network capacity is $\Theta(W \sqrt{n})$ under the arbitrary network model, where $n$ nodes are arbitrarily placed in a disk of unit area and the channel bandwidth is $W$. Knuth notation is used to express this result, where $f(n$ )$=\Theta[g(n)]$ means $f(n)$ and $g(n)$ have the same growth level.

According to these researches, many calculations for the upper and lower bounds of wireless ad hoc network capacity and methods to improve network transmission capacity and throughput capacity have been proposed. However, the omnidirectional transmission mode has several disadvantages such as it owns a large attenuation level, inefficient use of transmit power, and all nodes near a pair of communication nodes need to remain silent in order to transmit successfully and so on. In order to significantly improve the spatial multiplexing of wireless channels, thereby the capacity and throughput, directional antenna transmission technology was proposed. When using directional antennas, multiple pairs of nodes located near each other can transmit at the same time without mutual interference, which only depends on the directional characteristic of the directional 
antenna. The emergence of the adaptive multibeam array antenna transmission technology makes each node communicate with other nodes in multiple directions at the same time, which further improves the network communication capability.

A method of network capacity calculation when using directional transmission technology in wireless ad hoc networks was proposed in [2]; it came out that the antenna model was simplified to a sector-shaped antenna model that only considered the main lobe effect and ignored the side lobe effect. In this model, the directional working beam sector area was compared with the entire circular radiation range. The research in [3] was continued on the basis of [2]; it deduced the upper and lower bounds of network capacity in the case of singlehop and multihop communication. It indicated that the capacity increases as the number of hops increases when in a certain range and becomes smaller when the number of hops exceeds this range. In [4], a hybrid antenna model used in singlebeam mode was proposed. This model considers the influence of the side lobe effect on the network capacity when actual situations are taken into account and simplifies the effect as a calculation factor that could be inserted into the derivation process. Similarly, in [5], they put forward that the influence on capacity caused by multibeam could be converted into a gain factor which could be easily used in the derivation process as well. A more convergent lower bound of the capacity calculating method under arbitrary network model was put forward in [6]. In [7], the influence of self-interference caused by beamforming on network capacity is studied, which lead us to think about the difference between the side lobe effect and selfinterference. Different from the multibeam technology, Rahman et.al in [8] discussed from the perspective of multipacket reception to explore what kind of improvement their protocol will bring to network performance. In $[9,10]$, they mainly studied the improvement of network performance by cross-layer protocols and explored optimization strategies.

What is more, besides the discussion of the network capacity according to the node deployment method (that is, the protocol model in [1]), the analyses at the physical layer are also diverse. The research in [11] started with the power allocation in the physical layer, considered different power allocation modes for different transmission links, and analysed the optimal value of the network capacity under this condition. In [12], Zhao et al. considered the capacity of cellular network. From the perspective of another network model, the results could be mutually confirmed with the conclusion in [1]. The latest research achievements are focused on a more comprehensive theoretical model. In [13], a way was proposed to expand network capacity through MIMO technology when smart antennas are used; this is much like the multibeam technology to be analysed in our research. In [14], the use of millimeter wave multibeam transmission mode in the satellite layer network was considered to increase the overall network capacity, which can be mutually confirmed with the VANET capacity calculated by us. In [15-18], their researches are aimed at improving the performance of the network through the algorithm of resource scheduling and obtaining the maximum network capacity by assigning different bandwidths to different nodes. Research on the control of output power mode in order to pursue a more optimal network capacity is reflected in [19-22]; different nodes choose different transmit powers according to different task requirements, which can effectively improve the throughput of the topology.

When it comes to the analysis of the capacity in vehicular ad hoc networks, several researches have focused their efforts on this zone. In [19, 23-25], they investigate architecture and applications of the VANET in order to look for a relatively comprehensive vehicle connectivity agreement to ensure users' security.

However, when calculating the arbitrary network capacity, few predecessors have done the overall derivation of the multibeam technology; they just analyse single-beam mode comprehensively. In their researches, the analyses on the improvement of network capacity brought by directional transmission technology and multibeam technology are still in a very ideal state, and there are still many details that have not been considered in actual engineering applications.

Therefore, this paper mainly studies the network capacity of the VANET and analyses how much improvement the network capacity can be brought to by the use of multibeam directional transmission technology compared with the traditional antenna working mode. When computing the capacity, we fully take the influence of the directional antenna side lobe effect on the network capacity into account and derive the upper and lower bound of the arbitrary network capacity when the transceiver node pairs use the following four working modes. These working modes are omnidirectional transmission and omnidirectional reception, omnidirectional transmission and directional reception, directional transmission and omnidirectional reception, and directional transmission and directional reception. They will be abbreviated as OTOR, OTDR, DTOR, and DTDR in the rest of this paper. Finally, under normalized conditions, we compared the improvement of network capacity in several modes and found out the conditions that need to be satisfied in this case.

Our main contribution can be summarized as follows:

(1) We conduct a more in-depth study on the network capacity using directional multibeam technology and take the side lobe effect into account on the analysis of the wireless network capacity at the same time, which would make the results to have more realistic significance. Capacity analyses on different transmission model have been carried out as well

(2) We horizontally compare the convergence of the network capacity under different interference models and explore how to select the interference model if the upper and lower bounds of the wireless network capacity are sensitive to the various model parameters

(3) We longitudinally compare the changes in network capacity performance under different antenna modes and find the conditions which make the network capacity be maximized

The rest of the paper is organized as follows. In Section 2 , we will describe the models we built and parameters we 
should use, and then, we will explain their meanings. In Sections 3 and 4, we will discuss the upper and lower bounds of the arbitrary network model under two interference models (the protocol model and the physical model). In Section 5, we analyse the results obtained in the previous two sections. In Section 6, we will summarize and analyse our results and then describe future research directions.

\section{Parameters and Models}

In this section, meanings of all symbols and parameters used in the following derivation process will be explained and listed in Table 1. At the same time, in order to make the subsequent derivation concise, we make some reasonable assumptions to obtain a more convenient calculation process and they are as follows:

(1) There are $n$ vehicular nodes arbitrarily located in a disk of unit area on the plane. (The results carry over to any domain of unit area in $R^{2}$ which is the closure of its interior.) Each node can transmit $W$ bits per second over a common wireless channel

(2) All nodes transmit with the same transmitting power $P$, which would make the calculation process less complex

(3) The transmitting or receiving beam width in the multibeam transmission model is identical, that is, all nodes share the same kind of adaptive multibeam array antenna and the same antenna state

2.1. Antenna Model. Most of the existing studies on the capacity of directional transmission networks use the simplified sector-based directional antenna model. This model can simplify the calculation process, but it ignores the influence of the side lobe effect on the calculation results, which makes results somewhat idealized. The hybrid antenna model is referenced in [4]. This model takes the side lobe effect and the antenna directional characteristic into account and handles the complexity of the calculation process well. In our research, the multibeam directional transmission mode needs to change the directional sector range (the number of beams) in it. The model is shown in Figure 1.

The model in the figure is a hybrid model of omnidirectional and directional modes. The sector area with a larger radius than the circular area represents the main lobe direction, whose beam angle is $\theta$, and the remaining circular area is surrounded by side lobes and back lobes, where the radius of the circle and the sector represents the length of the omnidirectional and directional radiation, respectively. We define the parameter $s$ as the ratio of the radius of $G_{\text {side }}$ and $G_{\text {main }}$ in the hybrid antenna mode, which is generally less than 1 . The gain of the omnidirectional antenna is $G_{\text {side }}$ as well. It can be seen that nodes can simultaneously receive signals from transmitters in the circular area and the sector area.

2.2. Network Model. We use the arbitrary network model in the following derivation process. In the setting of arbitrary network model, there are $n$ nodes arbitrarily located in a region of unit area (e.g., $1 \mathrm{~m}^{2}$ ). Each node arbitrarily selects a target node for to communicate, and it sends data to the target node at an arbitrary transmission rate. Therefore, the data transmission flow is random, and these nodes can arbitrarily choose the transmission distance and power level.

2.3. Interference Model. In a wireless ad hoc network, if nodes are densely distributed to a certain extent, concurrent transmission will occur, which will cause mutual interference. Therefore, nodes need to be deployed separately to avoid conflicts in the cross area. For this reason, we assume that there are interference areas that can guarantee successful transmission. Relying on the protocol model and physical model proposed by Gupta et al. in [1] and the interference area theory of directional transmission proposed by $\mathrm{Yi}$ et al. [2], we made a certain degree of improvement on this basis to establish an interference area model based on the received signal.

2.3.1. Protocol Model. Suppose node $X_{i}$ transmits to a node $X_{j}$. Then, in order for this transmission to be successful when node $X_{k}$ simultaneously transmits over the same channel, this condition needs to be satisfied.

$$
\left|X_{k}-X_{j}\right| \geq(1+\Delta)\left|X_{i}-X_{j}\right|
$$

where $X_{i}, X_{j}, X_{k}$, and $X_{l}$ refer to the nodes themselves and their current positions. The constant $\Delta>0$ models situations where a guard zone is specified by the protocol to prevent a neighboring node from transmitting at the same time. It also allows for imprecision in the achieved range of transmissions.

2.3.2. Physical Model. On the premise that the receiver SINR threshold $\beta$ (minimum SINR) is specified, the condition for the node $X_{j}$ to successfully receive the data transmitted from the node $X_{i}$ is

$$
\operatorname{SINR}=\frac{P C\left(G_{t} G_{r} /\left|X_{i}-X_{j}\right|^{\alpha}\right)}{N+\sum_{\substack{k \in \Gamma \\ k \neq i}}\left(P C \cdot G_{t} G_{r} /\left|X_{k}-X_{j}\right|^{\alpha}\right)} \geq \beta,
$$

where $G_{t}$ means the gain of transmitting antennas and $G_{r}$ means the gain of receiving antennas. In most cases, $\beta \geq 1$.

2.3.3. Interference Area Description. Now, let us calculate the area of the interference region, which is an important part of the subsequent calculation work. As mentioned above, we derive the network capacity under four working modes: OTOR, OTDR, DTOR, and DTDR.

In Figure 2, compared to the OTOR working mode, the other three modes cover a longer distance in the beam direction and a larger interference area.

First, we define two parameters; the parameter $r$ mentioned before refers to the antenna radiation radius in the directional transmission mode; what is more, the parameter $\xi_{a}(a=1,2,3,4)$ indicates the ratio between the area of $A_{\mathrm{OO}}$ and other three modes. 
TABLE 1: Symbols and parameters' description.

\begin{tabular}{|c|c|}
\hline Symbols and parameters & Explanation \\
\hline$m$ & Number of beams \\
\hline$\lambda$ & Node transmission rate (bit/s) \\
\hline$\eta$ & The gain of multibeam \\
\hline$T$ & Total communication time \\
\hline$\Gamma$ & The set of all nodes transmitting in the same channel simultaneously \\
\hline C & A constant determined by antenna heights, wavelength, and so on \\
\hline$d$ & Euclidean distance between transmitting node and receiving node \\
\hline$\alpha$ & $\alpha \geq 2$ means path loss exponent \\
\hline$G_{\text {main }}$ & Main lobe gain \\
\hline$G_{\text {side }}$ & Side lobe gain \\
\hline$s$ & The ratio of the radius of $G_{\text {side }}$ and $G_{\text {main }}$ in the hybrid antenna mode \\
\hline$N$ & The ambient noise power level at the receiver \\
\hline$r(n)$ & Node communication radius \\
\hline $\bar{L}$ & Average distance from source to destination \\
\hline$\beta$ & The minimum signal-to-interference plus noise ratio (SINR) for successful receptions at the receiver \\
\hline$h(b)$ & Total number of hops required for the data-bit $b$ to move from source to destination \\
\hline
\end{tabular}

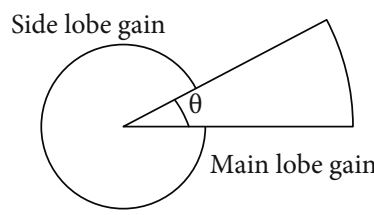

Single beam transmission

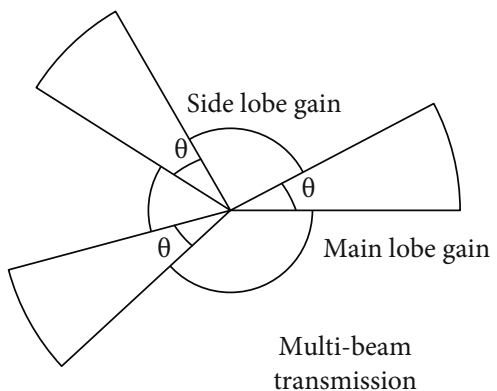

FIGURE 1: Hybrid antenna model in directional transmission mode.
For OTOR, the area of the interference region is

$$
A_{\mathrm{OO}}=\pi r_{1}^{2}=\pi r_{1}^{2} \xi_{1}
$$

where $r_{1}$ means the radius of the omnidirectional antenna radiation pattern.

For OTDR, the model is shown in Figure 2(a); taking the single-beam transmission mode, the area is

$$
A_{\mathrm{OD} 1}=\pi(s r)^{2}+\frac{\theta}{2 \pi}\left[\pi r^{2}-\pi(s r)^{2}\right]=\pi r^{2}\left[s^{2}+\left(1-s^{2}\right) \frac{\theta}{2 \pi}\right]=\pi r^{2} \xi_{2} .
$$

When the multibeam mode is adopted, since the number of beams directed by the receiving node to the transmitting node has not changed, the area has not changed.
For DTOR, the model is shown in Figure 2(b), and taking the single-beam transmission mode, the area is

$$
\begin{aligned}
A_{\mathrm{DO} 1}= & \pi r^{2} P\left\{\left|X_{i}-X_{j}\right| \leq s r\right\}+\pi r^{2} P\left\{\left|X_{i}-X_{j}\right|>s r\right\} \\
& \cdot P\left\{X_{i} \longrightarrow X_{j}\right\}=\pi r^{2}\left[s^{2}+\left(1-s^{2}\right) \frac{\theta}{2 \pi}\right]=A_{\mathrm{OD} 1} .
\end{aligned}
$$

Different from OTDR, the area has changed when adopting multibeam mode in that the number of the receiving beam has become $m$, and it changes to

$$
\begin{aligned}
A_{\mathrm{DO} m}= & \pi r^{2} P\left\{\left|X_{i}-X_{j}\right| \leq s r\right\}+\pi r^{2} P\left\{\left|X_{i}-X_{j}\right|>s r\right\} \\
& \cdot P\left\{X_{i} \longrightarrow X_{j}\right\}=\pi r^{2}\left[s^{2}+\left(1-s^{2}\right) \frac{m \theta}{2 \pi}\right]=\pi r^{2} \xi_{3} .
\end{aligned}
$$



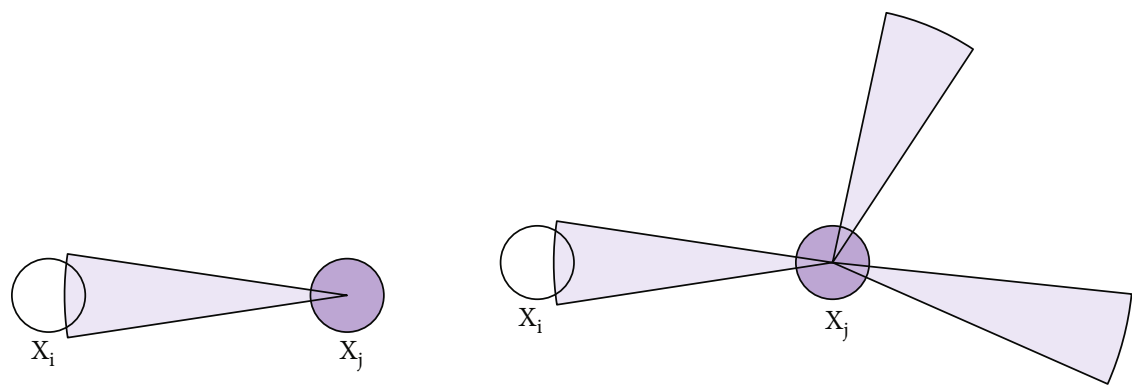

(a) The interference area of OTDR mode
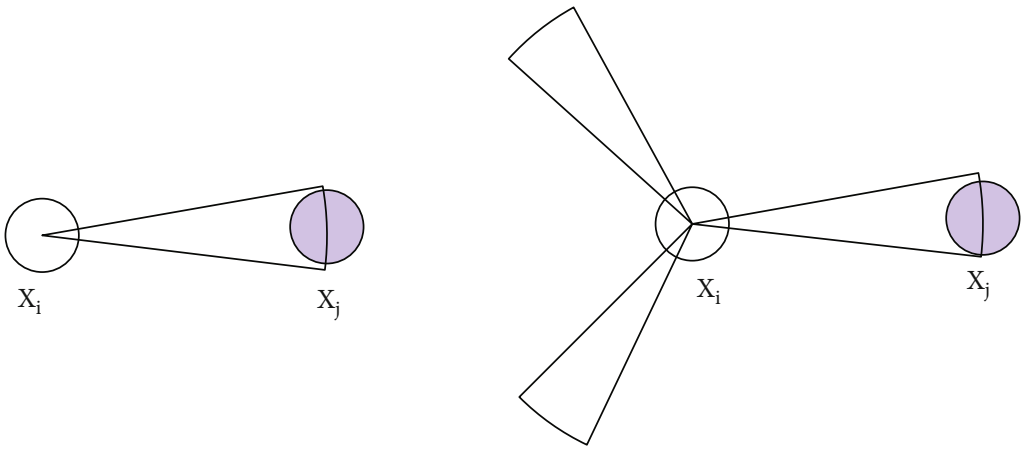

(b) The interference area of DTOR mode
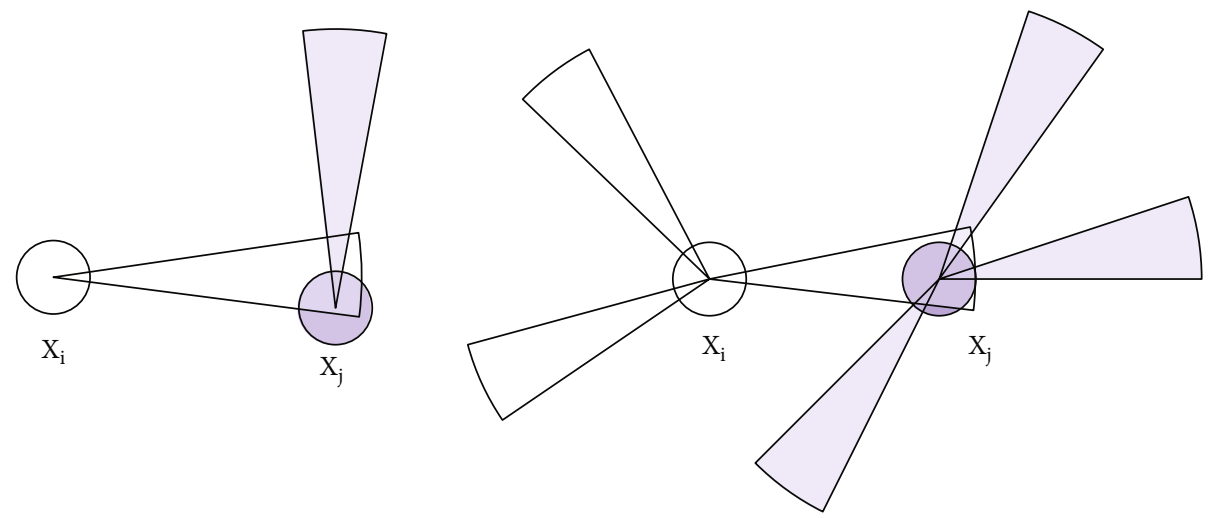

The transmitting area

The receiving area

(c) The interference area of DTDR mode

Figure 2: Interference area model.

For DTDR, the model is shown in Figure 2(c), and when taking the single-beam transmission mode, the area is

$$
\begin{aligned}
A_{\mathrm{DD} 1} & =\pi(s r)^{2}+\frac{\theta}{2 \pi}\left[\pi r^{2}-\pi(s r)^{2}\right] \cdot P\left\{T_{x} \longrightarrow R_{v}\right\} \\
& =\pi r^{2}\left[s^{2}+\left(1-s^{2}\right) \frac{\theta^{2}}{4 \pi^{2}}\right] .
\end{aligned}
$$

Similarly, after adopting multibeam transmission mode, the number of beams of the connected node pairs has changed, so the area has changed to

$$
\begin{aligned}
A_{\mathrm{DD} m} & =\pi(s r)^{2}+\frac{\theta}{2 \pi}\left[\pi r^{2}-\pi(s r)^{2}\right] \cdot P\left\{T_{x} \longrightarrow R_{v}\right\} \\
& =\pi r^{2}\left[s^{2}+\left(1-s^{2}\right) \frac{m^{2} \theta^{2}}{4 \pi^{2}}\right]=\pi r^{2} \xi_{4} .
\end{aligned}
$$

2.4. Multibeam Gain Description. Taking into account that when using multibeam technology, to achieve the optimal network capacity, the $n$ nodes in the network are divided into two groups: transmitting group and receiving group, where every receiving node receives from $m$ transmitting nodes, the maximum simultaneous transmission a receiver can handle. Assuming that the transmitting group has $x$ 
nodes, then the receiving group has $(n-x)$ nodes. In OTOR and DTDR modes, all nodes can be divided into two parts, half for transmission and half for reception. At this time, the OTOR gain equals to 1 , and the DTDR gain is $\eta=m$. In the OTDR mode, the transmit flow is equal to the receive flow, that is, $x W=(n-x) m W$; therefore, it can be concluded that the gain of network capacity at this time is $\eta=$ $((n m /(m+1)) W+(n-(n m /(m+1))) m W) / n W=2 m /(m$ $+1)$. Similarly, in the DTOR mode, it can be derived that the gain is equal to that in the OTDR mode.

\section{The Upper Bound of Arbitrary Network Capacity}

If the communication time is $T$ seconds and the node transmission rate is $\lambda$ bits per second, $\lambda n T$ bits of total data can be transmitted. If the average distance from source to destination is $L$, the whole topology can transmit $\lambda n L$ bit-meters per second.

In the following of this section, we may derive the upper bound of the network capacity based on the two interference models proposed in the above section.

3.1. Using the Protocol Model of the Interference. Supposing bit $b$, where $1 \leq b \leq \lambda n T$, is moving from its source to the destination with $h(b)$ hops in $T$ seconds, where the $h$ th hop traverses the distance of $r_{b}^{h}$. Since the transmission distance is at least equal to the total length of the path connecting the source with the destination, so we have

$$
\sum_{b=1}^{\lambda n T} \sum_{h=1}^{h(b)} r_{b}^{h} \geq \lambda n T L .
$$

Before the subsequent derivation process is carried out, we introduce a new parameter $H$, which means the total number of hops that all bits transmit in time $T$, i.e., $H=$ $\sum_{b=1}^{\lambda n T} h(b)$. Therefore, the number of bits transmitted by all nodes in $T$ seconds is equal to $H$. As mentioned in Section 2.4 , the gain in the multibeam mode is $\eta$, so

$$
H=\sum_{b=1}^{\lambda n T} h(b) \leq \frac{W T \eta n}{2} .
$$

According to $[1,4]$, we can get that all nodes communicate in the same subchannel and time will not interfere with each other if each hop consumes a disk of radius $\Delta / 2$ times the length of the hop around each receiver, i.e., $r_{b}^{h}$.

Considering the edge effect, if a neighbor node is close to the edge of the area and noting that a range greater than the diameter of the domain is unnecessary, at least a quarter of its interference circular area caused by the neighbor node is within the communication range. Within the same communication time period, at most $W T$ bits of data flow can be generated. Therefore, we can obtain that $\sum_{b=1}^{\lambda n T} \sum_{h=1}^{h(b)}(1 / 4)$ $(\Delta / 2)^{2} \cdot A_{I} \leq \eta W T$, and it can be rewritten as

$$
\sum_{b=1}^{\lambda n T} \sum_{h=1}^{h(b)} \frac{\left(r_{b}^{h}\right)^{2}}{H} \leq \frac{16 \eta W T}{\pi \xi_{a} \Delta^{2} \mathrm{H}} .
$$

Note that the quadratic function on the left side of inequality (9) is convex, so we can get

$$
\left(\sum_{b=1}^{\lambda n T} \sum_{h=1}^{h(b)} \frac{r_{b}^{h}}{H}\right)^{2} \leq \sum_{b=1}^{\lambda n T} \sum_{h=1}^{h(b)} \frac{\left(r_{b}^{h}\right)^{2}}{H}
$$

Combining expressions (9), (10), (11), and (12), the upper bound of the network capacity can be obtained as

$$
\lambda n T L \leq \frac{2 \eta W T}{\Delta} \sqrt{\frac{2 n}{\pi \xi_{a}}}=\left\{\begin{array}{l}
\frac{2 m W T}{\Delta} \sqrt{\frac{4 m W T}{\pi\left[s^{2}+\left(1-s^{2}\right)\left(m^{2} \theta^{2} / 4 \pi^{2}\right)\right]}} \text { (the capacity in DTDR mode), } \\
\frac{4 m W T}{\frac{2 n}{\pi\left[s^{2}+\left(1-s^{2}\right)(m \theta / 2 \pi)\right]}} \text { (the capacity in DTOR mode), } \\
\frac{W T}{\frac{(m+1) \Delta}{\pi\left[s^{2}+\left(1-s^{2}\right)(\theta / 2 \pi)\right]}} \text { (the capacity in OTDR mode), } \\
\frac{8 n}{\pi} \text { (the capacity in OTOR mode). }
\end{array}\right.
$$

Note that the result in OTOR mode is consistent with the result obtained in [1].

3.2. Using the Physical Model of the Interference. The difference from the protocol model is that in order to perform subsequent calculations in the physical model, inequality (9) needs to be replaced with different expressions. According to inequality (2), if the power of transmitting nodes is also put into the entire set of the same time slot and on the same subchannel, i.e., add the numerator on the left side 
of the inequality to the denominator, then (2) can be rewritten as

$$
\frac{P C\left(G_{t} G_{r} /\left|X_{i}-X_{j}\right|^{\alpha}\right)}{N+\sum_{k \in \Gamma}\left(P C \cdot G_{t} G_{r} /\left|X_{k}-X_{j}\right|^{\alpha}\right)} \geq \frac{\beta}{\beta+1} .
$$

Hence, we can get

$$
\left|X_{i}-X_{j}\right|^{\alpha} \leq \frac{\beta+1}{\beta} \cdot \frac{P C \cdot G_{t} G_{r}}{N+\sum_{k \in \Gamma}\left(P C \cdot G_{t} G_{r} /\left|X_{k}-X_{j}\right|^{\alpha}\right)} .
$$

Since the communication area is a disk of unit area, in other words, the area is 1 ; therefore, we can easily get $\pi$ $\left(\left|X_{i}-X_{j}\right| / 2\right)^{2} \leq 1,\left|X_{i}-X_{j}\right| \leq 2 / \sqrt{\pi}$. In addition, expression (15) can be rewritten as $\left|X_{i}-X_{j}\right|^{\alpha} \leq((\beta+1) / \beta) \cdot\left(\left(P C \cdot G_{t}\right.\right.$ $\left.\left.G_{r}\right) /\left(N+(\pi / 4)^{\alpha / 2} \cdot \sum_{k \in \Gamma} P C \cdot G_{t} G_{r}\right)\right)$.

While summing all the transmitting and receiving nodes in the same channel at this communicating time, we can get

$$
\begin{aligned}
\sum_{i \in \Gamma}\left|X_{i}-X_{j}\right|^{\alpha} & \leq \frac{\beta+1}{\beta} \cdot \frac{\sum_{i \in \Gamma} P C \cdot G_{t} G_{r}}{N+(\pi / 4)^{\alpha / 2} \cdot \sum_{k \in \Gamma} P C \cdot G_{t} G_{r}} \\
& =\frac{\beta+1}{\beta} \cdot \frac{1}{\left(N /\left(\sum_{i \in \Gamma} P C \cdot G_{t} G_{r}\right)\right)+(\pi / 4)^{\alpha / 2}\left(\sum_{k \in \Gamma} G_{t} G_{r} / \sum_{i \in \Gamma} G_{t} G_{r}\right)} \\
& \leq \frac{\beta+1}{\beta} \cdot\left(\frac{4}{\pi}\right)^{\alpha / 2} \frac{\sum_{i \in \Gamma} G_{t} G_{r}}{\sum_{k \in \Gamma} G_{t} G_{r}} .
\end{aligned}
$$

Due to different working modes, the antenna gains of the transmitting and receiving pairs are also different. Therefore, we use the parameter $\gamma_{a}$ to characterize the ratio $\sum_{i \in \Gamma} G_{t} G_{r}$ I $\sum_{k \in \Gamma} G_{t} G_{r}$ and it is

$$
\gamma_{a}=\frac{\sum_{i \in \Gamma} G_{t} G_{r}}{\sum_{k \in \Gamma} G_{t} G_{r}}=\left\{\begin{array}{l}
\frac{\sum_{i \in \Gamma} G_{\text {side }} G_{\text {side }}}{\sum_{k \in \Gamma} G_{\text {side }} G_{\text {side }}}=1 \text { (in OTOR mode) }, \\
\frac{\sum_{i \in \Gamma} G_{\text {side }} G_{\text {main }}}{\sum_{k \in \Gamma} G_{\text {side }} G_{\text {side }}}=\frac{1}{s}(\text { in OTDR mode }), \\
\frac{\sum_{i \in \Gamma} G_{\text {main }} G_{\text {side }}}{\sum_{k \in \Gamma} G_{\text {side }} G_{\text {side }}}=\frac{1}{s}(\text { in DTOR mode }), \\
\frac{\sum_{i \in \Gamma} G_{\text {main }} G_{\text {main }}}{\sum_{k \in \Gamma} G_{\text {side }} G_{\text {side }}}=\frac{1}{s^{2}}(\text { in DTDR mode }) .
\end{array}\right.
$$

Therefore, (13) can be rewritten as

$$
\sum_{i \in \Gamma}\left|X_{i}-X_{j}\right|^{\alpha} \leq \frac{\beta+1}{\beta} \cdot\left(\frac{4}{\pi}\right)^{\alpha / 2} \gamma_{a} .
$$

It can be seen from the previous analysis that $r_{b}^{h}=\mid X_{i}$ $-X_{j} \mid$. Therefore, by adding up the communication data stream in all subchannels of all time slots, an expression similar to inequality (9) can be obtained that

$$
\sum_{b=1}^{\lambda n T} \sum_{h=1}^{h(b)} \frac{\left(r_{b}^{h}\right)^{\alpha}}{H} \leq \frac{((\beta+1) / \beta) \cdot(4 / \pi)^{\alpha / 2} \gamma_{a} \eta W T}{H \xi_{a}} .
$$

And after the similar derivation process, we can get the capacity that

$$
\lambda n T \bar{L} \leq \frac{\eta W T}{\sqrt{\pi}}\left[\frac{(2 \beta+2) n^{\alpha-1} \gamma a}{\beta \xi a}\right]^{1 / \alpha}=\left\{\begin{array}{l}
\frac{m W T}{\sqrt{\pi}}\left[\frac{(2 \beta+2) n^{\alpha-1}}{\beta\left[s^{2}+\left(1-s^{2}\right)\left(m^{2} \theta^{2} / 4 \pi^{2}\right)\right] s^{2}}\right] \text { (the capacity in DTDR mode), } \\
\frac{2 m W T}{(m+1) \sqrt{\pi}}\left[\frac{(2 \beta+2) n^{\alpha-1}}{\beta\left[s^{2}+\left(1-s^{2}\right)(m \theta / 2 \pi)\right] s}\right] \text { (the capacity in DTOR mode), } \\
\frac{2 m W T}{(m+1) \sqrt{\pi}}\left[\frac{(2 \beta+2) n^{\alpha-1}}{\beta\left[s^{2}+\left(1-s^{2}\right)(\theta / 2 \pi)\right] s}\right] \text { (the capacity in OTDR mode), } \\
\frac{W T}{\sqrt{\pi}}\left[\frac{(2 \beta+2) n^{\alpha-1}}{\beta}\right] \text { (the capacity in OTOR mode). }
\end{array}\right.
$$

\section{The Lower Bound of the Arbitrary Network Capacity}

We mainly focus on the lower bound of network capacity by giving that the node throughput can be achieved in a specific scenario. Therefore, we fix the all pairs of transmitterreceiver combination, i.e., the direction of transmitting beam and receiving beam is determined and fixed, and different node deployment models are determined according to dif- ferent working modes. From the previous section, we know that the area of the whole communication region is 1 , which means the radius is $1 / \sqrt{\pi}$. Then, we establish a rectangular coordinate system on this circle, and the origin is the center of the circle.

4.1. Network Capacity in OTOR Mode. Just like Figure 3, we will place receivers at these locations: $(j(1+2 \Delta) r \pm \Delta r, k(1$ $+2 \Delta) r)$ and $(j(1+2 \Delta) r, k(1+2 \Delta) r \pm \Delta r)$, if $|j+k|$ is odd. 


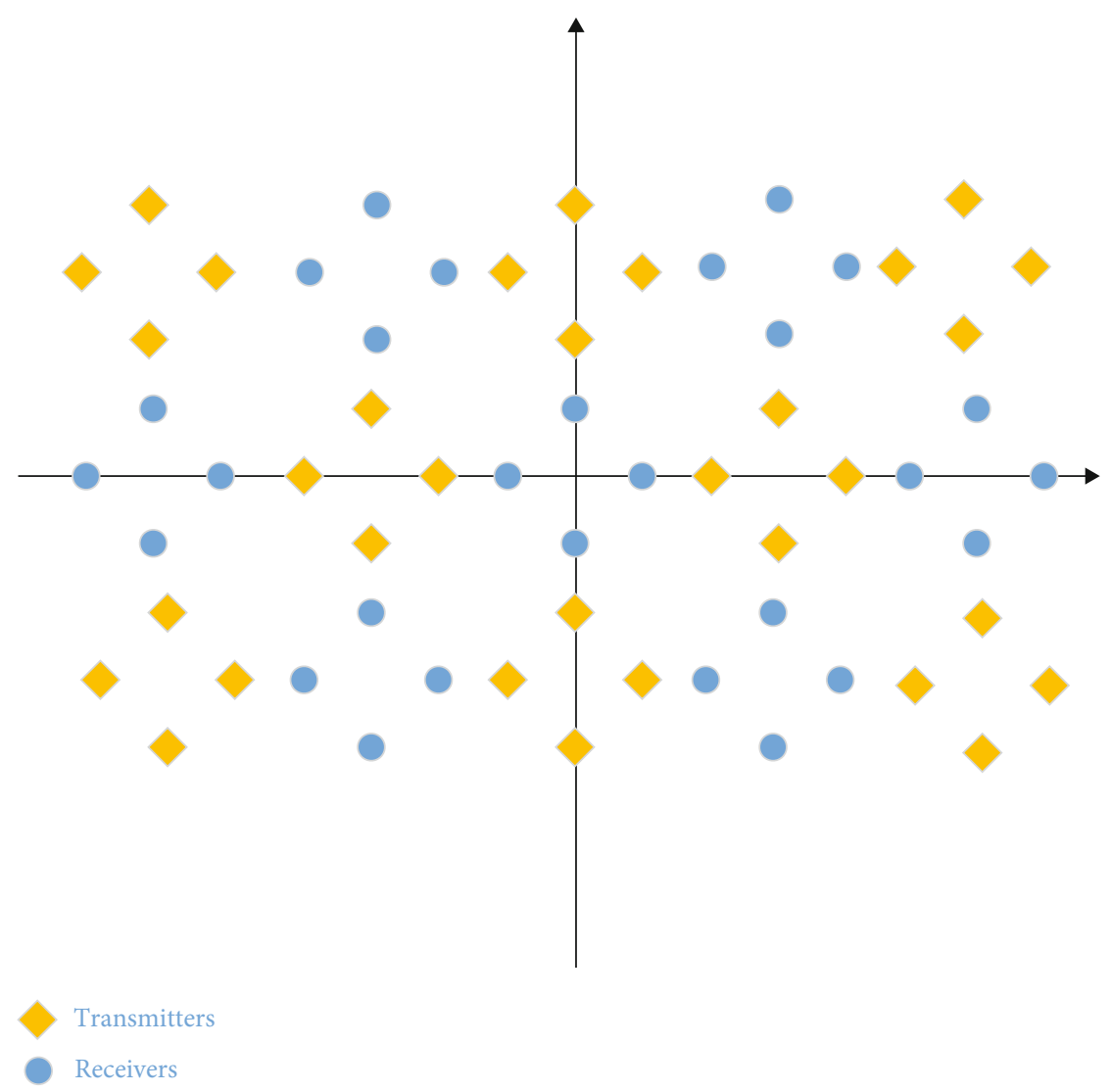

Figure 3: Node deployment model in OTOR mode.

Then, we place transmitters at these locations $(j(1+2 \Delta$ )$r \pm \Delta r, k(1+2 \Delta) r)$ and $(j(1+2 \Delta) r, k(1+2 \Delta) r \pm \Delta r)$ if $\mid j$ $+k \mid$ is even.

In the protocol model, all transmitters transmit to their nearest receiver, and the transmission distance is $r$. On this condition, there is no interfering node in the middle, and there will be $n / 2$ pairs of receivers and transmitters in the communication area. Now, we define a new parameter $L$, and $L=(1$ $+2 \Delta) r$, which means the side length of the square that nodes deployed on. So according to [3], all such squares that intersect with a disk of radius $((1 / \sqrt{\pi})-\sqrt{2} L)$ are entirely contained in the communication area; therefore, we can get that the number of these squares is at least $\left(\pi((1 / \sqrt{\pi})-\sqrt{2} L)^{2}\right) / L^{2}$. It can be observed from Figure 3 that each square contains two pairs of transmitting and receiving nodes; hence,

$$
\frac{\pi((1 / \sqrt{\pi})-\sqrt{2} L)^{2}}{L^{2}} \cdot 2=\frac{n}{2} .
$$

Then, we can derive that the communication radius is $r$ $=L /(1+2 \Delta)=2 /((1+2 \Delta)(\sqrt{8 \pi}+\sqrt{n}))$, and it is equal to the average distance between nodes according to our node's deployment strategy. Therefore, the capacity is

$$
\lambda n T L=W \frac{n}{2} T r=\frac{n W T}{(1+2 \Delta)(\sqrt{n}+\sqrt{8 \pi})} .
$$

Now, we turn to the physical model, according to expression (2) and description of the link interference in the physical model in [6], we give the relevant interference factor in path transmission, denoted by $a_{s}$, to be

$$
a_{s}=\beta \cdot \frac{N+\sum_{k \in \Gamma}\left(P C \cdot G_{t} G_{r} /\left|X_{k}-X_{j}\right|^{\alpha}\right)}{P C\left(G_{t} G_{r} /\left|X_{i}-X_{j}\right|^{\alpha}\right)}=\beta\left(1+\frac{1}{\operatorname{SINR}}\right)=\beta \frac{S+I+N}{S} \text {, }
$$

where $S=P C\left(G_{t} G_{r} /\left|X_{i}-X_{j}\right|^{\alpha}\right)$ means the transmitted signal power. Let $c=(1+2 \Delta)$. It can be seen from Figure 3 that the distance between any two transmitters $\left(S_{i}, S_{j}\right)$ is not less than $(c-1)$ times the distance between the nearest transceiver pair $\left(S_{i}, R_{i}\right)$, that is, $d_{\left(S_{i}, S_{j}\right)} \geq(c-1) d_{\left(S_{i}, R_{i}\right)}=(c-1) r$.

Therefore, the circles of radius $((c-1) r) / 2$ around the transmitter do not intersect each other. We call the concentric circles formed by nodes placed at the same distance from the center of the transmitter as $\operatorname{Ring}_{k}$, where $k=0,1,2 \cdots$. If $k$ $=0$, it means that there is no transmitter. The circle with radius $((c-1) r) / 2$ around the transmitter also belongs to this level of concentric circle. The area of the $k$ th concentric ring is

$$
\begin{aligned}
A\left(\operatorname{Ring}_{k}\right) & =\pi\left\{\left[(k+1) c r+\frac{(c-1) r}{2}\right]^{2}-\left[k c r-\frac{(c-1) r}{2}\right]^{2}\right\} \\
& =\pi c(2 c-1)(2 k+1) r^{2} .
\end{aligned}
$$


Therefore, the interference caused by concentric rings at each level is

$$
\begin{aligned}
I_{k} \leq \sum_{S_{w} \in \operatorname{Ring}_{k}} I_{S_{w}} & \leq \frac{A\left(\operatorname{Ring}_{k}\right)}{\pi[((c-1) r) / 2]^{2}} \cdot \frac{P}{(k c r)^{\alpha}} \\
& \leq \frac{4 P c(2 k+1)(2 c-1)}{c^{\alpha} r^{\alpha} k^{\alpha}(c-1)^{2}} \leq \frac{2^{5} 3 P}{c^{\alpha} r^{\alpha} k^{\alpha-1}},
\end{aligned}
$$

where $S_{w}$ indicates one node belongs to Ring $k_{k}$.

Summing all interferences in the concentric rings, we get

$$
I_{\text {sum }} \leq \sum_{k=1}^{\infty} I_{k} \leq \sum_{k=1}^{\infty} \frac{2^{5} 3 P}{c^{\alpha} r^{\alpha} k^{\alpha-1}}<\frac{\alpha-1}{\alpha-2} \frac{2^{5} 3 P}{c^{\alpha} r^{\alpha}} .
$$

Bring inequality (18) into equation (23), we can get

$$
a_{s}=\beta\left(1+\frac{1}{\operatorname{SINR}}\right)=\beta \frac{S+I_{\text {sum }}+N}{S}<\beta \frac{I_{\text {sum }}}{S} \leq \frac{\alpha-1}{\alpha-2} \frac{2^{5} 3 \beta}{c^{\alpha}} .
$$

Therefore, we can obtain $\operatorname{SINR} \geq 1 /(((\alpha-1) /(\alpha-2))($ $\left.\left.96 / c^{\alpha}\right)-1\right)=1 /\left(((\alpha-1) /(\alpha-2))\left(96 /(1+2 \Delta)^{\alpha}\right)-1\right), \quad$ and since $\operatorname{SINR}_{\min }=\beta$, additionally we can get

$$
(1+2 \Delta)_{\max }=\left(\frac{96 \beta}{\beta+1} \frac{\alpha-1}{\alpha-2}\right)^{1 / \alpha} .
$$

Combining equation (28) with the expression of capacity, we can derive that the lower bound of the capacity in physical model is

$$
\lambda n T L=W \frac{n}{2} \operatorname{Tr}=\frac{n W T}{((96 \beta /(\beta+1))((\alpha-1) /(\alpha-2)))^{1 / \alpha}(\sqrt{n}+\sqrt{8 \pi})} .
$$

4.2. Network Capacity in OTDR Mode. First of all, we will still derive the lower bound of the capacity in the protocol model. Some parameters and their definitions ought to be clarified in that they will be reused. Let $r_{1}=\Delta r / 2, r_{2}=r+$ $r_{1}$, and $L=4 r_{2}$. Just like Section 4.1 , we place transmitting nodes at these locations: $\left[p L+\left(r+r_{1}\right) \cos ((\pi / 2)+((2 i-1)\right.$ $\left./ m) \pi), q L+\left(r+r_{1}\right) \sin ((\pi / 2)+((2 i-1) / m) \pi)\right]$, and place receiving nodes at these locations: $\left[p L+r_{1} \cos ((\pi / 2)+((2 i\right.$ $\left.-1) / m) \pi), q L+r_{1} \sin ((\pi / 2)+((2 i-1) / m) \pi)\right]$, where $p, q$, $i$, and $j$ are integers and $i \in[0, m-1], j \in[0, m / 6]$. The node deployment strategy is shown in Figure 4.

Similar to the derivation process in the above section, the number of transceiver pairs arranged in the square is changed to $m$ on this condition; therefore, its communication radius should also change to $r=1 /((4+2 \Delta)(\sqrt{2 \pi}+\sqrt{n / 2 m}$ ) ), and the capacity in this mode is

$$
\begin{aligned}
\lambda n T L= & \frac{n}{2} \eta \sqrt{\xi_{a}} r W T \\
= & \frac{n m W T}{(m+1)(2+\Delta)} \\
& \cdot \frac{1}{\sqrt{s^{2}+\left(1-s^{2}\right)(\theta / 2 \pi)}(\sqrt{8 \pi}+\sqrt{2 n / m})} .
\end{aligned}
$$

Now, we turn to the physical model; the derivation process is carried on according to expression (23) as well. Let parameter $c$ change into $c=(4+2 \Delta)$. While we do not need to change the area calculation method of each concentric ring level, the interference in each level that needs to consider further is the directional gain, i.e.,

$$
\begin{aligned}
I_{k} \leq \sum_{S_{w} \in \operatorname{Ring}_{k}} I_{S_{w}} & \leq \frac{A\left(\operatorname{Ring}_{k}\right)}{\pi[((c-1) r) / 2]^{2}} \cdot \frac{P G_{\text {main }}}{(k c r)^{\alpha}} \\
& \leq \frac{4 P c(2 k+1)(2 c-1) G_{\text {main }}}{c^{\alpha} r^{\alpha} k^{\alpha}(c-1)^{2}} \leq \frac{2^{5} 3 P G_{\text {main }}}{c^{\alpha} r^{\alpha} k^{\alpha-1}} .
\end{aligned}
$$

Therefore, the corresponding changes need to be taken into consideration, and finally, we can obtain the lower bound of the capacity in this mode that

$$
\begin{aligned}
\lambda n T L= & \frac{n}{2} \eta{\sqrt{\xi_{a}}}_{r} W T \\
= & \frac{n m W T}{(m+1)\left(\left(96 G_{\text {main }} \beta /(\beta+1)\right)((\alpha-1) /(\alpha-2))\right)^{1 / \alpha}} \\
& \cdot \frac{1}{\sqrt{s^{2}+\left(1-s^{2}\right)(\theta / 2 \pi)}(\sqrt{8 \pi}+\sqrt{2 n / m})} .
\end{aligned}
$$

4.3. Network Capacity in DTOR Mode. We can obtain the same conclusion by swapping the transmitter and receiver positions in Section 4.2, and the result is similar to expressions (30) and (32); they are

$\lambda n T L=\frac{n}{2} \eta \sqrt{\xi_{a}} r W T=\left\{\begin{array}{l}\frac{n m W T}{(m+1)(2+\Delta)} \cdot \frac{1}{\sqrt{s^{2}+\left(1-s^{2}\right)(m \theta / 2 \pi)}(\sqrt{8 \pi}+\sqrt{2 n / m})} \text { (if in the protocol model), } \\ \frac{n m W T}{(m+1)\left(\left(96 G_{\text {main }} \beta /(\beta+1)\right)((\alpha-1) /(\alpha-2))\right)^{1 / \alpha}} \cdot \frac{1}{\sqrt{s^{2}+\left(1-s^{2}\right)(m \theta / 2 \pi)}(\sqrt{8 \pi}+\sqrt{2 n / m})} \text { (if in the physical model). }\end{array}\right.$ 


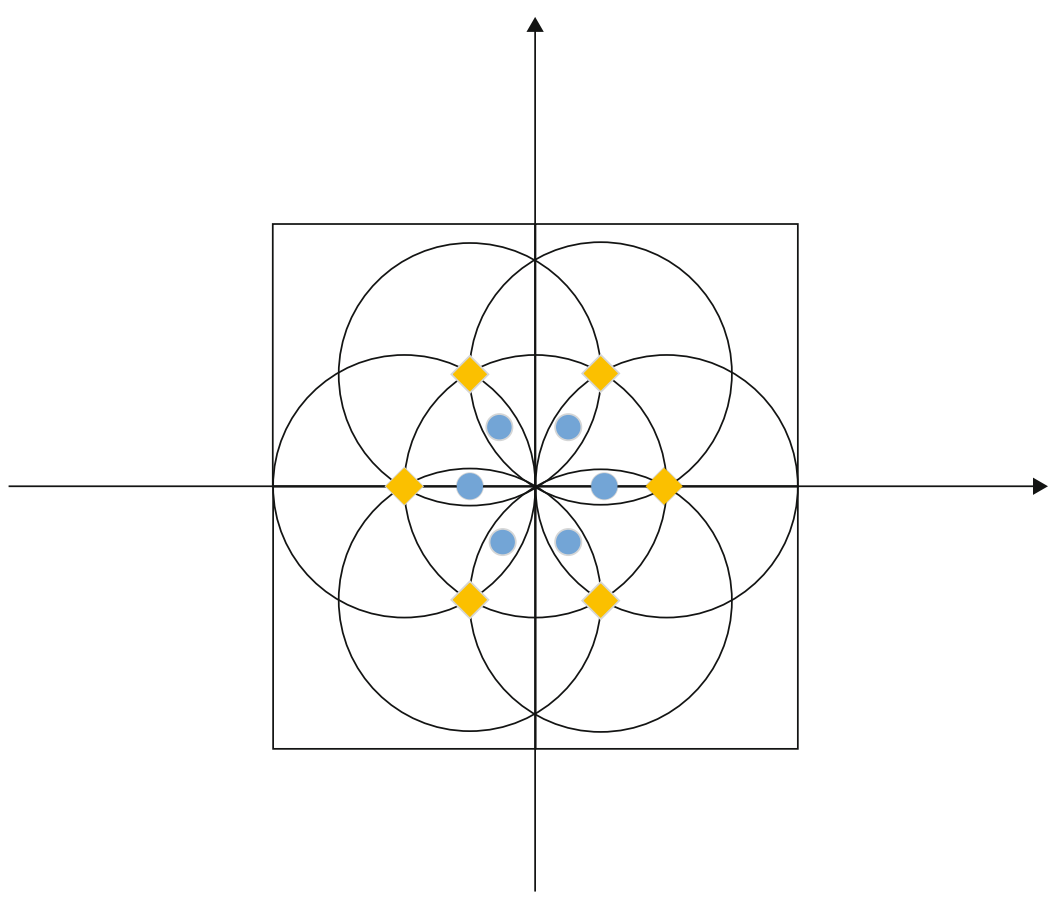

Transmitters

Receivers

FIgURE 4: Node deployment model on the condition of $m=6$ in OTDR mode.

4.4. Network Capacity in DTDR Mode. Similarly, we first consider the lower bound of the capacity in the protocol model. We need to change the side length of the square to $L=2(1+\Delta) r$, and let $r_{3}=\Delta r / 4, r_{4}=r+r_{3}$. After that, we place transmitting nodes in the following positions: $[p L+$ $r_{3} \cos ((\pi / 2)+((2 i-1) / m) \pi), q L+r_{3} \sin ((\pi / 2)+((2 i-1) /$ $m) \pi)]$ and $\left[p L+(L / 2) \cos (((3 m+4 i \pm 4 j-2) / 2 m) \pi)+r_{3}\right.$ $\cos (((m+4 i-2) / m) \pi), q L+(L / 2) \sin (((3 m+4 i \pm 4 j-2) /$ $\left.2 m) \pi)+r_{3} \sin (((m+4 i-2) / m) \pi)\right]$. At the same time, receiving nodes are arranged in the following positions: $[p L$ $+r_{4} \cos ((\pi / 2)+((2 i-1) / m) \pi), q L+r_{4} \sin ((\pi / 2)+((2 i-$ $1) / m) \pi)]$ and $\left[p L+(L / 2) \cos (((3 m+4 i \pm 4 j-2) / 2 m) \pi)+r_{4}\right.$ $\cos (((m+4 i-2) / m) \pi), q L+(L / 2) \sin (((3 m+4 i \pm 4 j-2) / 2$ $\left.m) \pi)+r_{4} \sin (((m+4 i-2) / m) \pi)\right]$. The node deployment strategy is shown in Figure 5.

Similarly, the nodes are arranged in a square with a side length of $L$, and $y=m \times(2+(\lfloor m\rfloor / 3))$ pairs of receiving and transmitting nodes are arranged in this square area. Therefore, $\left(\pi((1 / \sqrt{\pi})-\sqrt{2} L)^{2}\right) / L^{2} \cdot y=n / 2$.

At this time, we get $L=1 /(\sqrt{n / 2 y}+\sqrt{2 \pi})=2(1+\Delta) r$. Therefore, the node's communication radius is $r=1 /(2(1+$ $\Delta)(\sqrt{n / 2 y}+\sqrt{2 \pi})$, and the capacity is

$$
\begin{aligned}
\lambda n T L= & \frac{n}{2} \eta r \sqrt{\xi_{a}} W T=\frac{n m W T}{2(1+\Delta)} \\
& \cdot \frac{1}{\sqrt{s^{2}+\left(1-s^{2}\right)\left(m^{2} \theta^{2} / 4 \pi^{2}\right)}(\sqrt{n /((m / 2)\lfloor m / 3\rfloor+m)}+\sqrt{8 \pi})} .
\end{aligned}
$$

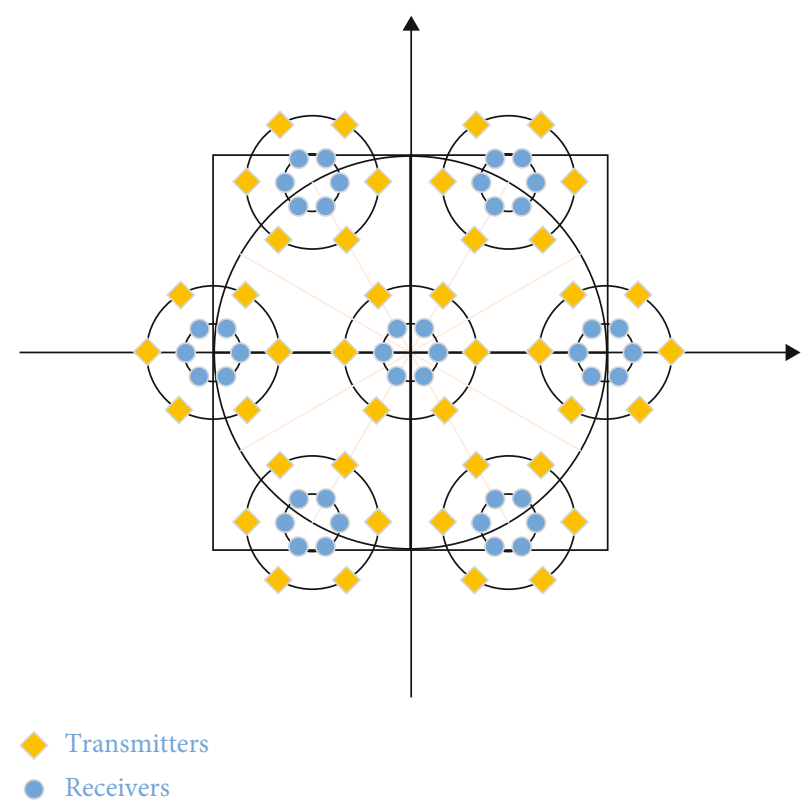

FIgURE 5: Node deployment model on the condition of $m=6$ in DTDR mode.

Now, we turn to the physical model; we need to change the parameter $c$ to $c=2+2 \Delta$ at first, and the following derivation is guided by the calculation process of expression (27). While the area calculation of the concentric rings does not need to be changed as well, the number of transmitting beams and receiving beams of each level has the corresponding increase when considering the gain from multibeam 


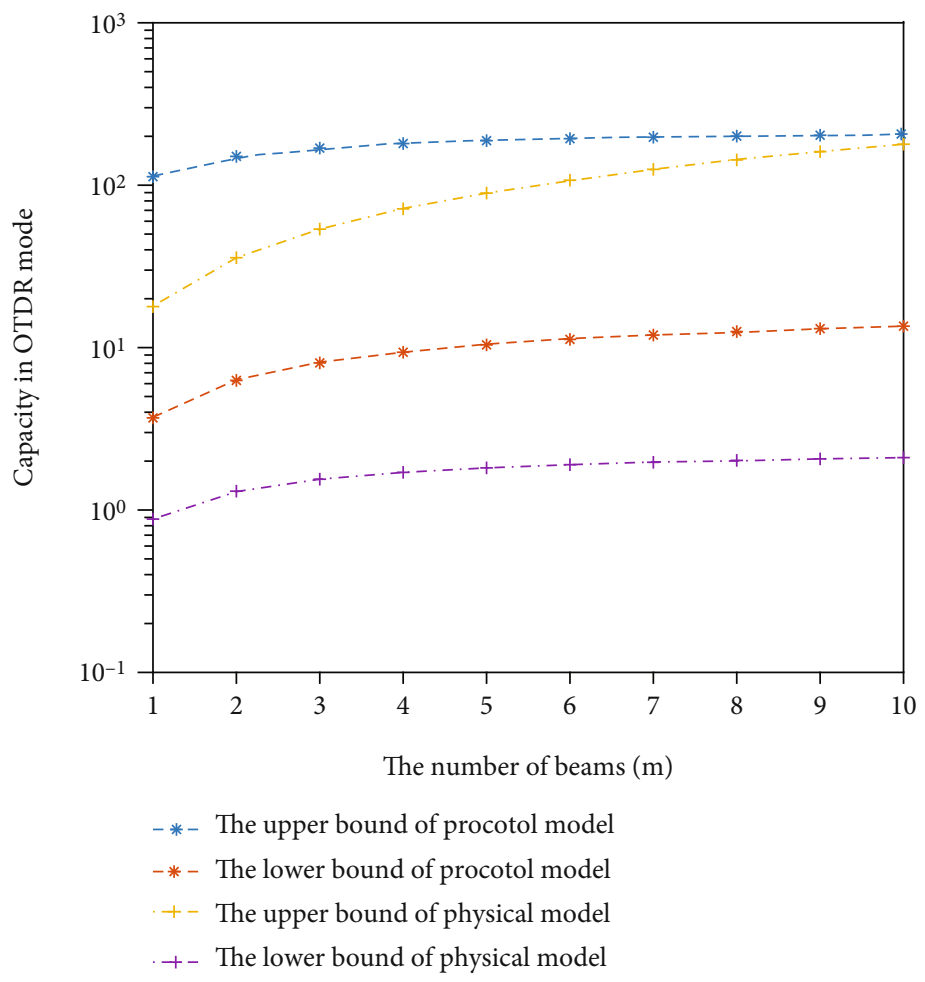

FIgURE 6: The upper and lower bounds in OTDR mode.

technology. Therefore, the number of transmitted signals in each level of the ring also needs to be changed. What is more, on the condition that the number of transmit beams and the directional gain of the transmitting and receiving have changed, the interference of each level also needs to be changed to

$$
\begin{aligned}
I_{k} \leq \sum_{S_{w} \in \operatorname{Ring}_{k}} I_{S_{w}} & \leq \frac{A\left(\operatorname{Ring}_{k}\right)}{\pi[((c-1) r) / 2]^{2}} \cdot \frac{m P G_{\text {main }}^{2}}{(k c r)^{\alpha}} \\
& \leq \frac{4 P m c(2 k+1)(2 c-1) G_{\text {main }}^{2}}{c^{\alpha} r^{\alpha} k^{\alpha}(c-1)^{2}} \\
& \leq \frac{2^{5} 3 m P G_{\text {main }}^{2}}{c^{\alpha} r^{\alpha} k^{\alpha-1}} .
\end{aligned}
$$

The subsequent derivation is similar to the process in Section 4.1 , so the capacity is

$$
\begin{aligned}
\lambda n T L= & \frac{n}{2} \eta r \sqrt{\xi_{a}} W T \\
= & \frac{n m W T}{\left(\left(96 \beta m G_{\text {main }}^{2} /(\beta+1)\right)((\alpha-1) /(\alpha-2))\right)^{1 / \alpha}} \\
& \cdot \frac{1}{\sqrt{s^{2}+\left(1-s^{2}\right)\left(m^{2} \theta^{2} / 4 \pi^{2}\right)}(\sqrt{n /((m / 2)\lfloor m / 3\rfloor+m)}+\sqrt{8 \pi})} .
\end{aligned}
$$

\section{Mathematical Analysis}

5.1. Convergence under Different Interference Models. In order to make the calculated results more meaningful and comparative, we randomly assigned values to the parameters. In order to make the calculated value have universal meaning, our calculation process will be carried out under normalized conditions. Given $n=100, W=1(\mathrm{~Hz}), T=1$ (s), $\theta=\pi / 6, \alpha=3, \beta=1.5, \Delta=0.5$, and $s=0.2$, we set the number of beams $m$ as a variable and calculate the upper and lower bounds of the four modes by making $m$ from 1 to 10 , respectively. The results are shown in Figures 6-8. We find that on these conditions the gain for capacity in OTDR and DTOR modes is relatively small, while the gain for capacity in DTDR mode has to be significantly improved. In addition, the protocol model is more convergent than the physical model.

This phenomenon has caught our attention. Does the network capacity obtained by the protocol model really be more convergent than the physical model under any circumstances? Hence, we operate the analysis by making all the parameters as variables and compare these results in a two-dimensional rectangular coordinate system. From Figures 6-8, we can know when $m \geq 6$, the change in network capacity is small. From Figure 7, we can know the maximum of capacity under the protocol model when $m=2$. At the same time, this is in line with our expectations. In DTOR mode, for the sake that the more beams transmitted, the greater the interference to the receiving node, so the overall network capacity will decrease 


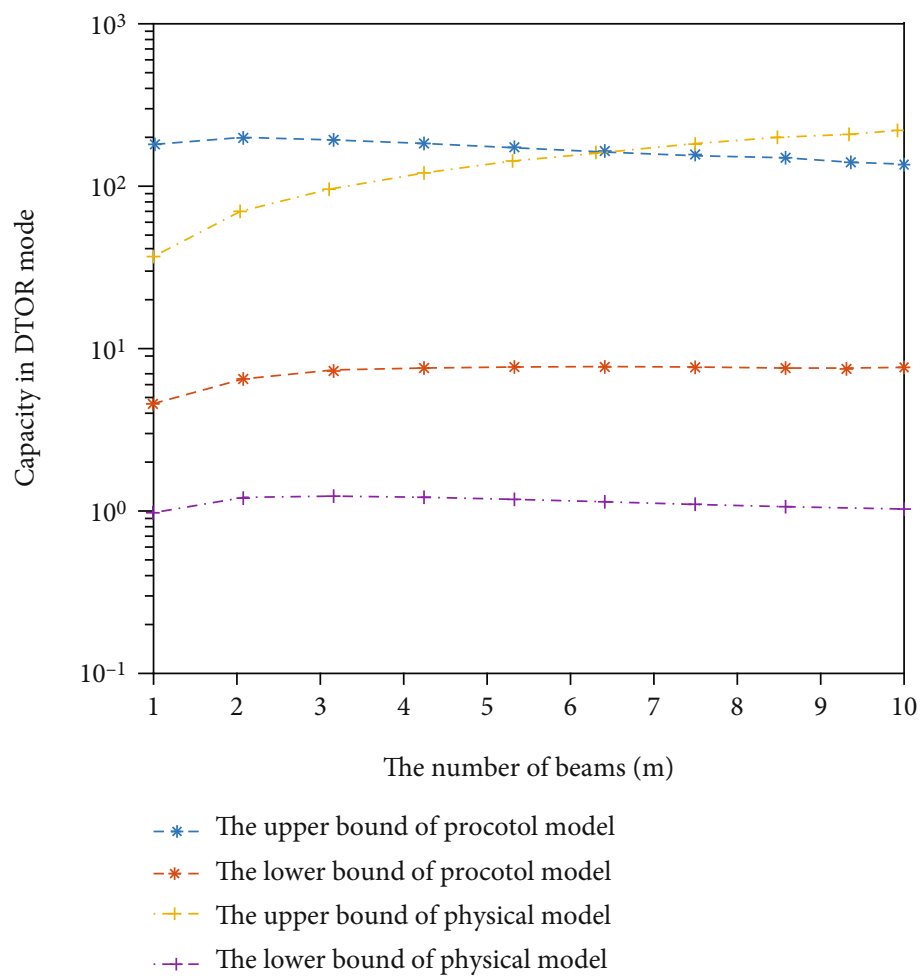

FIgURE 7: The upper and lower bounds in DTOR mode.

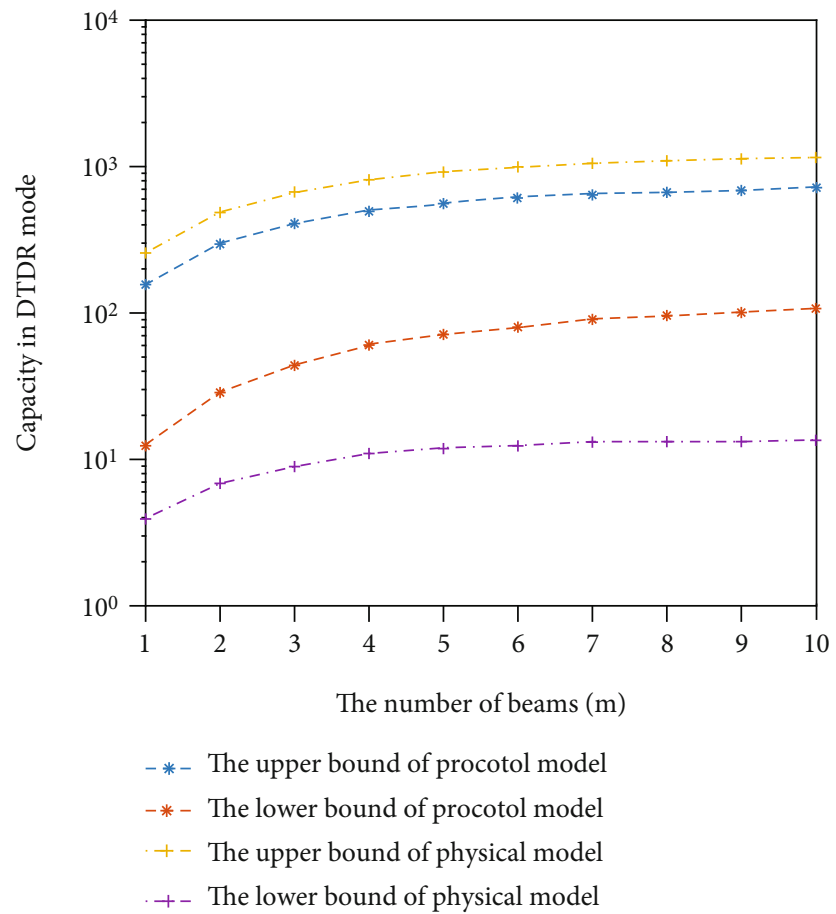

FIgURE 8: The upper and lower bounds in DTDR mode.

as the number of waves increases. Therefore, taking all the above and the actual situation into account, we take $m=6$ as the benchmark value. Since all the calculation results can be replaced as $\Theta(W T \sqrt{n})$ when they are converted to scales, we turn our attention to $\alpha, \beta$, and $\Delta$ and use them as variables to find the law among capacity changes.

In order to make our models can be compared under a unified standard, we look for the internal connections between 


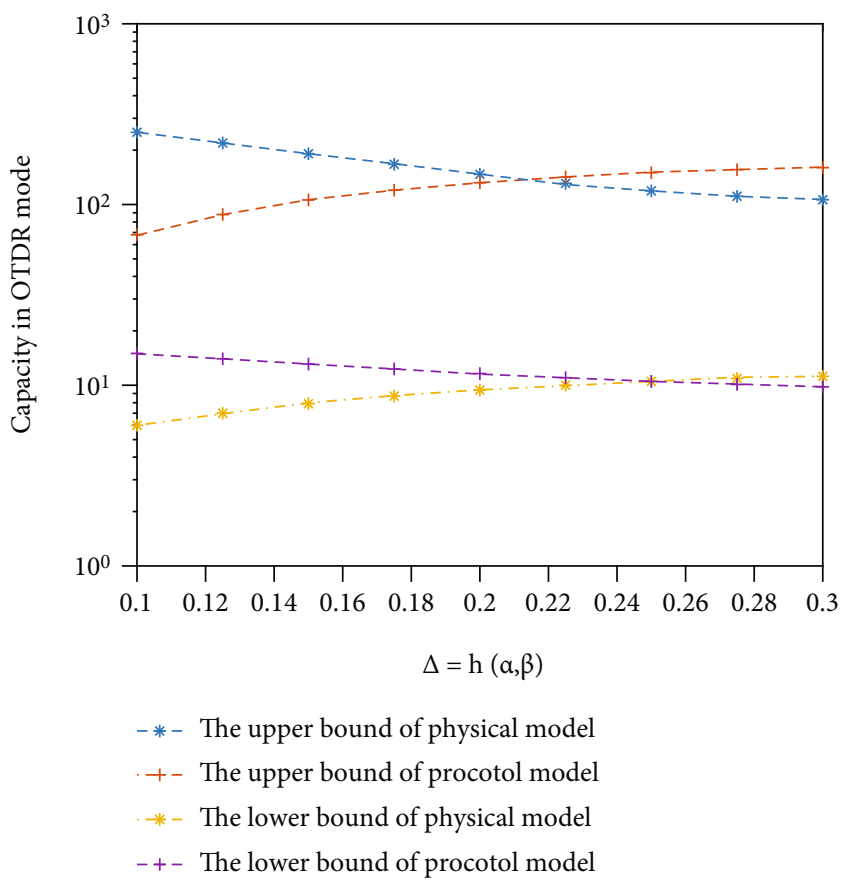

FIGURE 9: Comparison of convergence between two interference models in OTDR mode.

variables. In the derivation process, we found that there is a mutual transformation relationship between $\Delta$ and $\alpha, \beta$. Therefore, we use the parameter $\Delta$ in the protocol model as a reference variable to look for changes of capacity in different interference models.
The analysis of the upper bound is first carried out. We compare the capacity of the physical model when $2<\alpha \leq 5$ and $1<\beta \leq 3$ with the capacity of the protocol model when $0.1 \leq \Delta \leq 0.3$, and the results are shown in Figures 9-11. We use

$$
\Delta=h(\alpha, \beta)=\left\{\begin{array}{l}
\left.\left(\frac{\beta P_{\min }}{(\beta+1) P_{\max }}\right)^{1 / \alpha}-1 \text { (in all upper bound calculations }\right), \\
\left(\frac{96 \beta G_{\text {main }}(\alpha-1)}{(\beta+1)(\alpha-2)}\right)^{1 / \alpha}-2(\text { in the lower bound of OTDR and DTOR mode }), \\
\left.\left(\frac{96 \beta G_{\text {main }}^{2}(\alpha-1)}{(\beta+1)(\alpha-2)}\right)^{1 / \alpha}-1 \text { (in the lower bound of DTDR mode }\right),
\end{array}\right.
$$

to represent the transformation relationship between $\alpha, \beta$ and $\Delta$, and the proof of correlation between parameters can be referenced in $[1,3,6,9]$. It is found that in the OTDR and DTDR mode when $\Delta \leq 0.22$, using the physical model can make the calculated value more convergent and when $\Delta \leq 0.26$ in the DTOR mode the physical model can make the calculated value more convergent. In other cases, it is the protocol model that makes the derived theoretical value of capacity more convergent.

The next is the analysis of the lower bound. It can be seen that in the OTDR and DTDR mode when $\Delta \geq 0.24$ using the physical model can make the calculated value more convergent and in the DTOR mode when $\Delta \geq 0.22$ the physical model can make the calculated value more convergent.
In actual application, due to the influence of the transmission medium and the electromagnetic environment, the path transmission loss $\alpha$ is generally a certain constant, and the radius of the protected area will not exceed 0.3 times the communication radius, i.e., $\Delta \leq 0.3, d \leq(1+0.3) r$. And because we usually find the maximum network capacity in actual engineering, based on the above analysis, we conclude that when $\Delta \leq 0.23$, we try to choose the protocol model as the measurement method; otherwise, we choose the physical model.

\subsection{Capacity Improvement Based on Directional Multibeam} Transmission. For the evaluation of network performance improvement, we only analyse the upper bound of the network capacity. We investigate the protocol model and the 


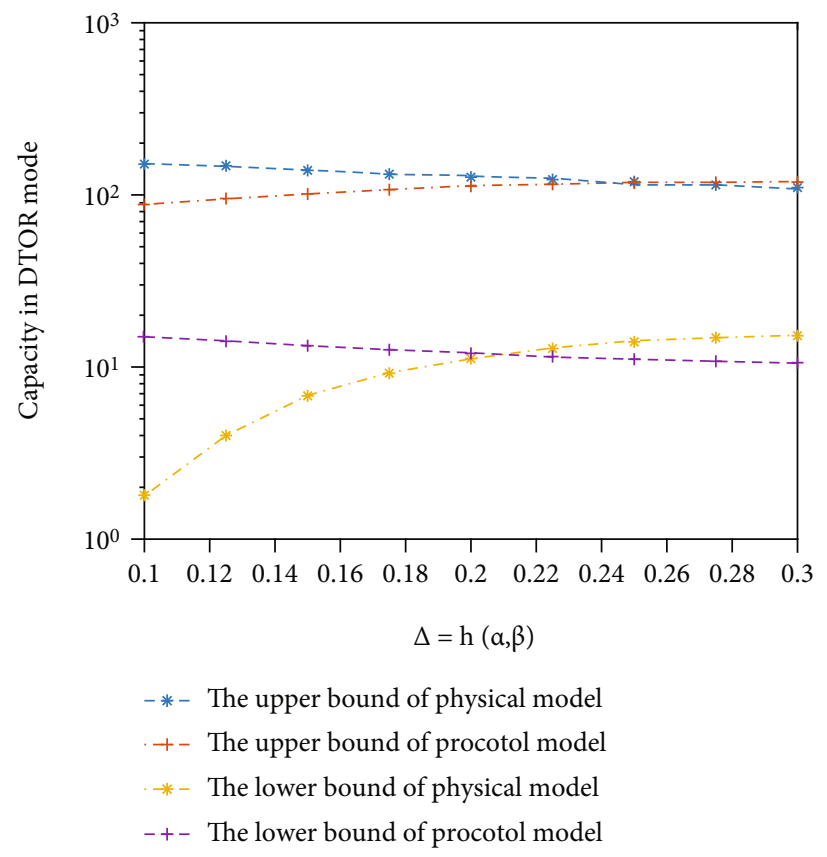

FIGURE 10: Comparison of convergence between two interference models in DTOR mode.

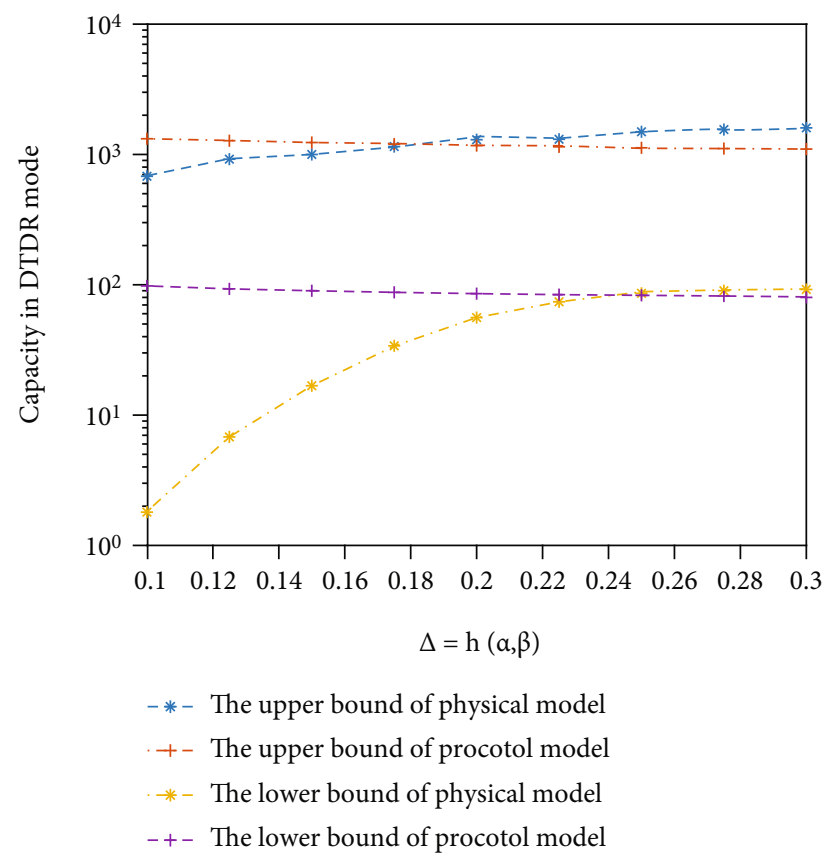

FIGURE 11: Comparison of convergence between two interference models in DTDR mode.

physical model separately and compare the changes in the network capacity when the number of beams changes.

According to the investigation in the above section, we set $\Delta=0.23, \alpha=2.5, \beta=1.5$ to discover the law among them, and the results are shown in Figures 12 and 13.

From the figure, we can illustrate that even when the number of beams $m=1$, compared with the other two working modes, the improvement of network capacity by DTDR mode is more prominent, and the multibeam mode does have a considerable improvement over the single-beam mode. Compared with the DTOR mode, the OTDR mode has less interference at the receiving node, which leads to a larger increase in network capacity. Different from the protocol model, the network capacity changes more smoothly under the physical model, which shows that, when the topology model is an arbitrary network model, it is easier to find general conclusions by analysing the network performance from the physical layer perspective. 


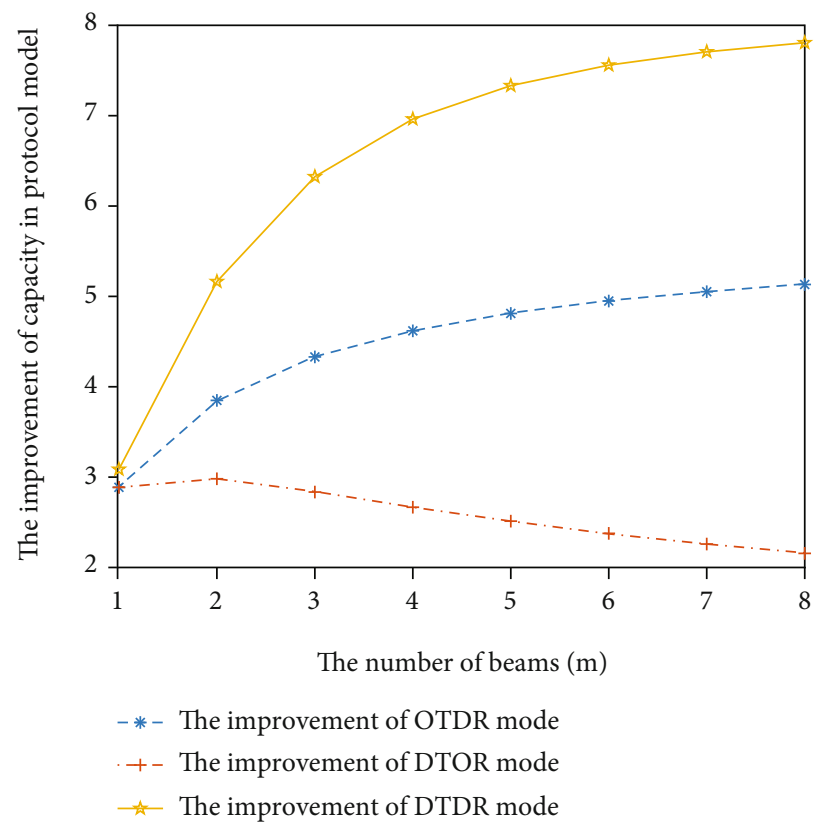

FIGURE 12: The improvement of network capacity by different working modes under the protocol model.

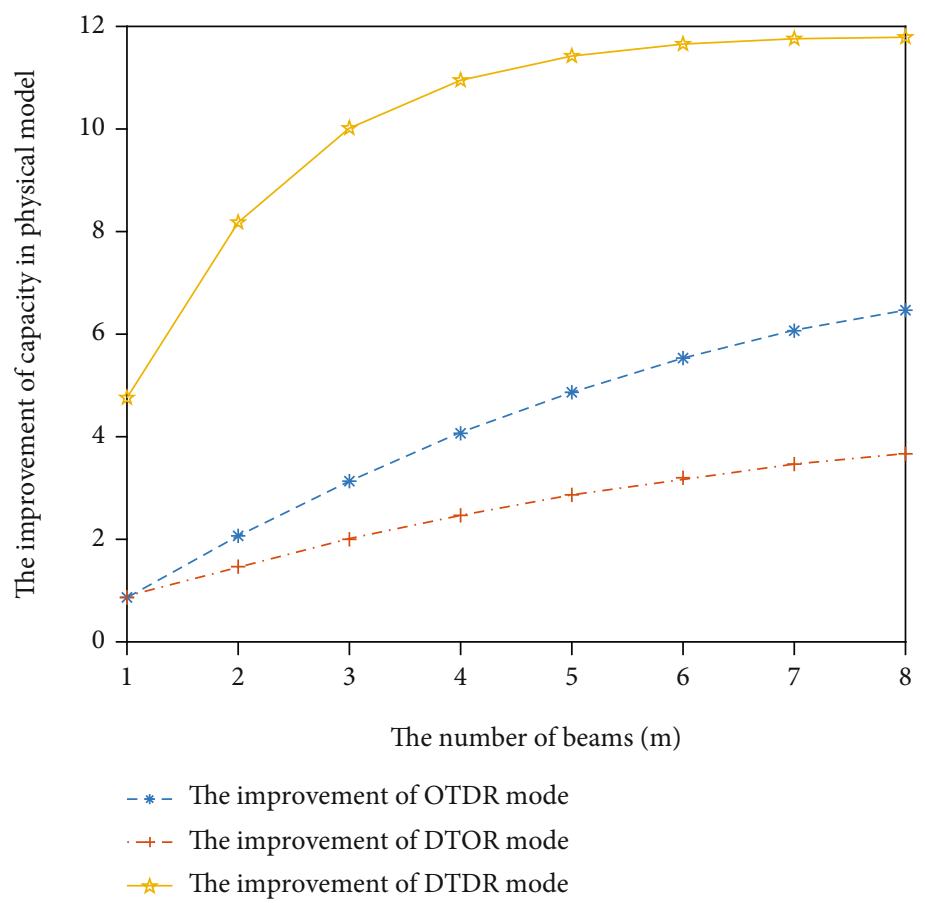

FIGURE 13: The improvement of network capacity by different working modes under the physical model.

5.3. Capacity Optimization Strategy. The purpose of our analysis for the network capacity improvement degree is to achieve the goal that capacity optimized to the maximum by adjusting the equipment from the VANET node in most scenarios. Since the path loss index $\alpha$ is fixed, the number of beams $m$ is determined by the beam angle $\theta$, the only two parameters that can be adjusted are the beam angle $\theta$ and the receiver SINR threshold $\beta$. Therefore, we study the rela- tionship between capacity and these two parameters, and the results are shown in Figures 14 and 15.

Since the receiver SINR threshold $\beta$ is only reflected in the capacity expression of the physical model, we only analyse the relationship between the network capacity and $\beta$ under the physical model. Therefore, when studying the relationship between beam angle and capacity, we only analyse the situation in the protocol model. As we can get from 


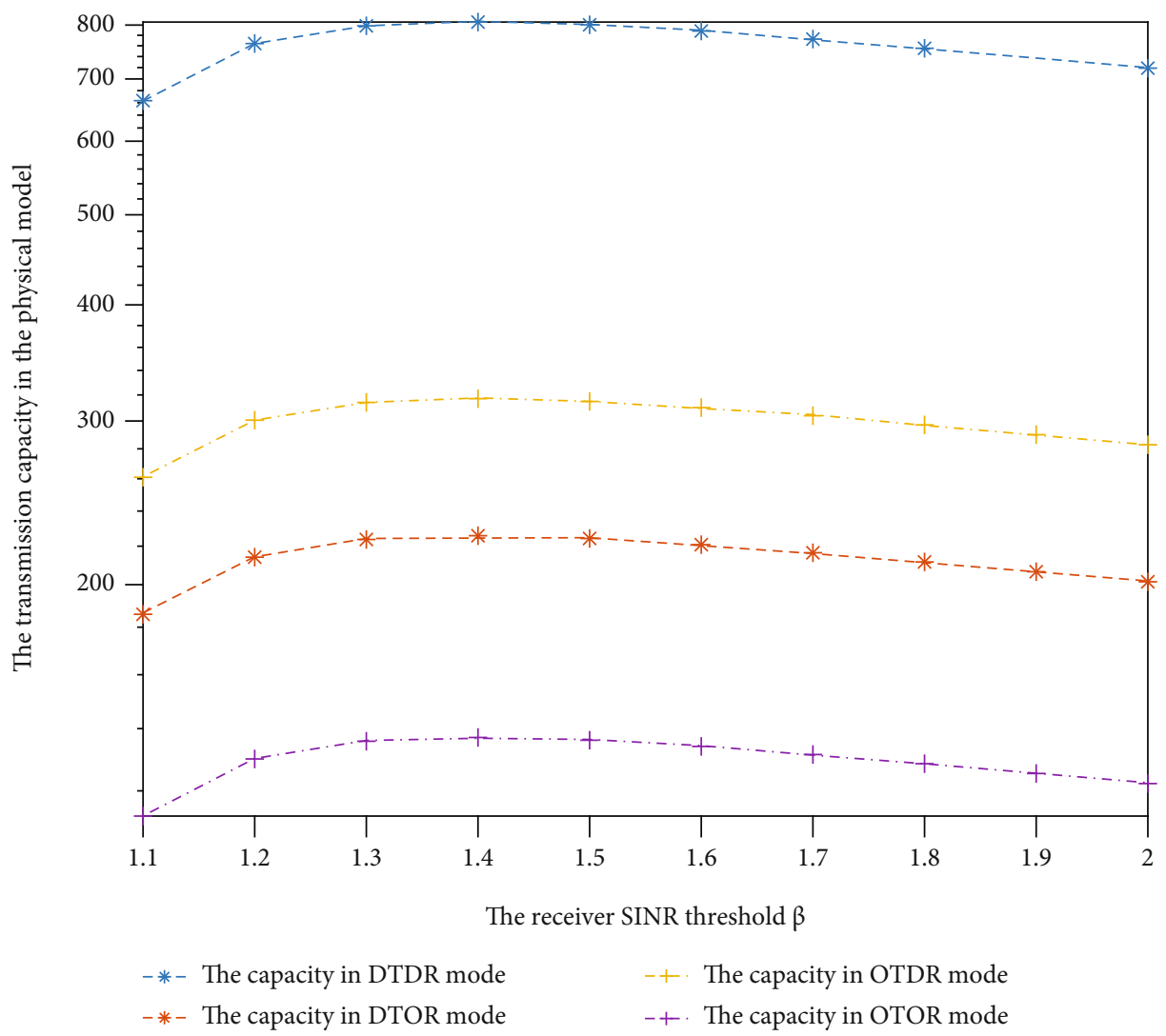

FIgURE 14: The relationship between the receiver SINR threshold $\beta$ and the VANET capacity.

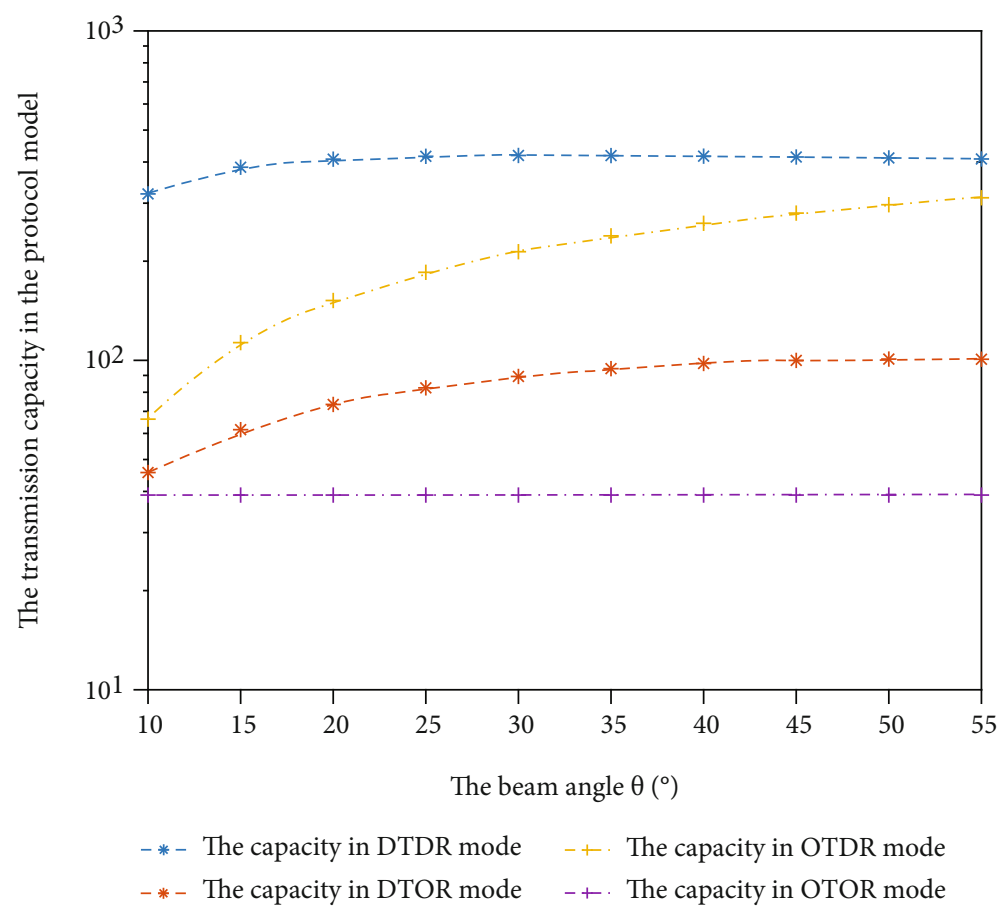

FIGURE 15: The relationship between the beam angle and the VANET capacity. 
Figure 14, the size of the capacity does not change linearly with the change of the parameter $\beta$. It achieves an extreme value at $\beta=1.4$, which shows that the increase of the receiver's SINR threshold will inevitably eliminate part of the signal energy.

Next, we observe Figure 15, and it can be seen that when the beam angle is in the range of $\left(25^{\circ}, 30^{\circ}\right)$, the network capacity in the DTDR mode is maximized. Therefore, the reason why we usually set the beam angle to $\theta=\pi / 6$ is that it can achieve the purpose of maximum capacity while being convenient to set up.

\section{Conclusions}

This paper mainly analyses the capacity under the vehicular ad hoc network based on multibeam directional transmission and derives the upper and lower bounds of the capacity in different working modes. Then, the convergence of the capacity under three working modes has been compared. Due to the actual conditions of the VANET, the guard zone coefficient $\Delta$ may not exceed 0.3 . Therefore, the protocol model may be chosen if $\Delta \leq 0.23$ and the physical model shall be chosen in other conditions. When considering the improvement of the capacity, multibeam transmission could bring greater network capacity improvement compared with single-beam transmission, and the change in network capacity tends to stabilize with the increase in the number of beams. When the wave number $m$ reaches a certain level, the improvement of the network capacity by the DTDR mode is 2 to 3 times that of the other two working modes.

Our future work will focus on establishing a unified interference model and network model, which will greatly simplify our calculation results, making the calculation of network capacity no longer so complicated and lengthy. Then, we may integrate the conclusions in this article to draw a more comprehensive network capacity result, which provides theoretical support for the subsequent cross-layer resource allocation of network capacity and the algorithm for finding the best channel allocation.

\section{Data Availability}

All the data can be calculated and analysed by readers who read this paper.

\section{Conflicts of Interest}

The authors declare that there is no conflict of interest regarding the publication of this paper.

\section{Acknowledgments}

This work is supported by the National Natural Science Foundation of China (No. 61671471).

\section{References}

[1] P. Gupta and P. R. Kumar, "The capacity of wireless networks," in IEEE Transactions on Information Theory, vol. 46, no. 2, pp. 388-404, 2000.
[2] S. Yi, Y. Pei, S. Kalyanaraman, and B. Azimi-Sadjadi, "How is the capacity of ad hoc networks improved with directional antennas?"” Wireless Networks, vol. 13, no. 5, pp. 635-648, 2007.

[3] P. Li, C. Zhang, and Y. Fang, "The capacity of wireless ad hoc networks using directional antennas," in IEEE Transactions on Mobile Computing, vol. 10, no. 10, pp. 1374-1387, 2011.

[4] J. Wang, L. Kong, and M. Wu, "Capacity of wireless ad hoc networks using practical directional antennas," in 2010 IEEE Wireless Communication and Networking Conference, pp. 16, Sydney, NSW, Australia, 2010.

[5] R. Wang, X. Wang, T. Chow et al., "Capacity and performance analysis for adaptive multi-beam directional networking," in MILCOM 2006-2006 IEEE Military Communications conference, pp. 1-7, Washington, DC, USA, 2006.

[6] O. Goussevskaia, R. Wattenhofer, M. M. Halldorsson, and E. Welzl, "Capacity of arbitrary wireless networks," in IEEE INFOCOM, pp. 1872-1880, Rio de Janeiro, Brazil, 2009.

[7] G. Yang and M. Xiao, "Performance analysis of millimeterwave relaying: impacts of beamwidth and self-interference," in IEEE Transactions on Communications, 2017.

[8] T. Rahman, H. Ning, and H. Ping, "DPCA: data prioritization and capacity assignment in wireless sensor networks," IEEE Access, vol. 5, 2017.

[9] Y. Wang, Q. Wang, H.-N. Dai, H. Wang, and Z. Shi, "Connectivity of cognitive radio ad hoc networks with directional antennas," Wireless Networks, vol. 24, no. 8, pp. 3045-3061, 2018.

[10] X. Jiang, H. Shokri-Ghadikolaei, G. Fodor et al., "Low-latency networking: where latency lurks and how to tame it," Proceedings of the IEEE, vol. 107, 2019.

[11] X. Zhang, H. Gong, and M. Liu, "Lower bounds on the capacity of wireless ad hoc networks," in 2016 International Wireless Communications and Mobile Computing Conference (IWCMC), pp. 714-718, Paphos, Cyprus, 2016.

[12] P. Zhao, L. Feng, P. Yu, W. Li, and X. Qiu, “A fairness resource allocation algorithm for coverage and capacity optimization in wireless self-organized network," China Communication, vol. 15, no. 11, pp. 10-24, 2018.

[13] R. Vaze and S. K. Iyer, "Capacity of cellular wireless network," in 201715th International Symposium on Modeling and Optimization in Mobile Ad Hoc, and Wireless Networks (WiOpt), pp. 1-8, Paris, 2017.

[14] G. O. Melih and D. Mubeccel, "Asymptotically throughput optimal scheduling for energy harvesting wireless sensor networks," IEEE Access, vol. 6, pp. 45004-45020, 2018.

[15] C. Jiang, Y. Shi, Y. T. Hou, W. Lou, S. Kompella, and S. F. Midkiff, "A general method to determine asymptotic capacity upper bounds for wireless networks," Network Science and Engineering, IEEE Transactions on, vol. 6, no. 1, pp. 2-15, 2019.

[16] C. Jiang and X. Zhu, "Reinforcement learning based capacity management in multi-layer satellite networks," IEEE Transactions on Wireless Communications, vol. 19, no. 7, pp. 46854699, 2020.

[17] Q. Cao, H. Rutagemwa, F. Zhou et al., "Capacity enhancement for mmwave multi-beam satellite terrestrial backhaul via beam sharing," in 2018 IEEE International Conference on Communications (ICC), pp. 1-6, Kansas City, MO, USA, 2018.

[18] S. Oda, M. Bouda, O. Vassilieva, Y. Hirose, T. Hoshida, and T. Ikeuchi, "Network capacity improvement by quality of 
transmission estimator with learning process," in 2017 European Conference on Optical Communication (ECOC), Gothenburg, Sweden, 2017.

[19] X. Ma, H. Lu, J. Zhao, Y. Wang, J. Li, and M. Ni, "Comments on interference-based capacity analysis of vehicular ad hoc networks," IEEE Communications Letters, vol. 21, no. 10, pp. 2322-2325, 2017.

[20] Z. Wei, Z. Wang, X. Yuan, H. Wu, and Z. Feng, "Information density-based energy-limited capacity of ad hoc networks," International Journal of Distributed Sensor Networks, vol. 14, no. 4, 2018.

[21] B. Yang, Z. Wu, Y. Fan, X. Jiang, and S. Shen, "Non-asymptotic capacity study in multicast mobile ad hoc networks," IEEE Access, vol. 7, pp. 115109-115121, 2019.

[22] S. Haddad and O. Leveque, On the Broadcast Capacity of Large Wireless Networks at Low SNR, IEEE, 2015.

[23] K. Raissi and B. B. Gouissem, "Hybrid communication architecture in VANETs via named data network," International Journal of Communication Systems, vol. 34, no. 11, 2021.

[24] T. Yeferny and S. Hamad, "Vehicular ad-hoc networks: architecture, applications and challenges," International Journal of Computer Science and Network Security, vol. 20, no. 2, 2020.

[25] S. More, R. Sonkamble, U. Naik, S. Phansalkar, P. More, and B. S. Saini, "Secured communication in vehicular adhoc networks (VANETs) using blockchain," IOP Conference Series: Materials Science and Engineering, vol. 1022, no. 1, article 012067, 2021. 